\title{
Parents Who Have Lost a Child to Cancer: What Do They Really Need?
}

\author{
Cécile Flahault ${ }^{1,2}$, Etienne Seigneur ${ }^{2,3}$, Valérie Laurence ${ }^{4}$, Hélène Pacquement ${ }^{3}$, \\ Sébastien Montel ${ }^{5}$ \\ ${ }^{1}$ Department of Psychology, Laboratory of Psychopathology and Health Process, \\ University of Paris Descartes, Boulogne Billancourt, France \\ ${ }^{2}$ Psycho-Oncology Department, Institut Curie, Paris, France \\ ${ }^{3}$ Pediatric Oncology Department, Institut Curie, Paris, France \\ ${ }^{4}$ Medical Oncology Department Institut Curie, Paris, France \\ ${ }^{5}$ Laboratory of Psychopathology and Neuropsychology, University Paris 8, Saint-Denis, France \\ Email: cecile.flahault@parisdescartes.fr
}

Received 20 March 2015; accepted 28 April 2015; published 5 May 2015

Copyright (C) 2015 by authors and Scientific Research Publishing Inc.

This work is licensed under the Creative Commons Attribution International License (CC BY).

http://creativecommons.org/licenses/by/4.0/

(c) (i) Open Access

\begin{abstract}
In a sample of parents who lost a child to cancer, we investigated their needs before and after their child's death and how these needs were fulfilled. We met parents of AYA (adolescents and young adults) between 15 and 25 years old, who died in Curie Institute between 2000 and 2003 . Twentyone families agreed to participate in this study between October 2005 and April 2006. When a family agreed to participate, a semi-structured interview was held in the psycho-oncology unit of the Curie Institute. This interview was audiotaped and then retranscribed for analysis. Data were subjected to the Grounded theory method, a systematic and standardized method of semantic data analysis. Three prominent themes emerged from our thematic data analysis: first, the importance of psychological care during the terminal phase of the child's disease and after the child's death, reported by $95 \%$ of parents; second, the need to be listened to and to be understood by the medical team ( $97 \%$ of parents); third, the need for information from the medical team. A big discrepancy was observed between psychological support expectations and the proportion of parents who actually consulted or participated in bereavement groups. Our study clearly demonstrates that parents who lose a child through cancer have particular needs and therefore deserve special attention.
\end{abstract}

\section{Keywords}

Cancer, Needs, Parents, Adolescents and Young Adults, Bereavement 


\section{Introduction}

The loss of a child is critical and traumatic for parents. Parents whose children have died from cancer live in a context of chronic uncertainty and apprehension (Monterosso \& Kristjanson, 2008). However, parental bereavement as well as their needs just before and after the child's death remains poorly understood.

A recent study explored the experience and needs of nine parents who had received hospital-based bereavement support following the death of their child from cancer (DeCinque et al., 2006). Parents identified the need for more supportive contacts from hospital staff during the palliative phase and following the child's death, early access to information on how to practically and emotionally prepare for the death of their child, contact with other bereaved parents and formal grief support for siblings.

In a study examining parental perspectives regarding bereavement services from a pediatric oncology treating center, D'Agostino et al. interviewed 7 parents whose children had died from cancer (D'Agostino et al., 2008). Parents perceived flexible and continuous bereavement services from the treating hospital as a necessary transition to community services. Talking to other parents with similar experiences and maintaining contact with staff at the treating hospital were considered critical for their healing after the loss.

Researchers emphasized that parents who had lost a child from cancer had a strong need to maintain contact with the team that cared of their children for months (Clerici et al., 2006). They observed that, in the process of coping with bereavement, anxiety and depression symptoms were common and not necessarily pathological, though there might be psychopathological reactions that could interfere with the parents' quality of life, when these symptoms are particularly intense or long-lasting. Several studies investigated risk factors associated with psychological distress. For instance, $\mathrm{Li}$ et al. underlined that the risk of mental disorders increased among parents, especially mothers, who lost a child ( $\mathrm{Li}$ et al., 2005). Concerning long term grief reactions, regarding the place of death and the gender of the parent, authors showed than one year after the child's death, fathers reported higher levels of psychological disturbance (depression, anxiety, and stress) when the child died at the hospital (Goodenough et al., 2004). On the other hand, there was no difference in the mother's psychological state as a function of the place of death.

Main psychological negative outcomes seemed to reveal complicated grief. Thus, identifying the needs of parents who have lost a child suffering from cancer is essential in order to preserve their quality of life.

We retrospectively asked 21 parents about their needs before and after their child's death and how these needs were fulfilled. The data presented here are part of a broader study on the place of death of adolescents and young adults (AYA) with cancer (Montel et al., 2008). This research is the first known parental bereavement study in oncology conducted in France, thereby extending (not just replicating) previous research findings to another country/culture (Barrera et al., 2009; Christ et al., 2003). On the other hand, AYA constitutes a unique group whose bereaved parents may have needs that differ from bereaved parents of younger or older children (Kavanaugh, Trier, \& Korzec, 2004). The expectations and needs for support of bereaved parents after caregiving have not been extensively and empirically investigated in France. This severe lack of knowledge hinders the implementing of relevant and refined support care for this vulnerable population. A broader international literature aims at capturing berefts' experience, trying to make their voice heard. In order to ensuring respect ethical imperatives while conducting bereavement research, authors suggested to favour qualitative studies with indepth interviews (Beck \& Konnert, 2007; Dyregrov, 2004; Hynson et al., 2006). Qualitative methodology allows addressing experimental themes while giving possibility for bereaved parents to make sense of their loss.

\section{Methods}

\subsection{Participants}

We contacted by mail and phone all parents of AYA died in the Curie Institute between 2000 and 2003. We consecutively met the 21 parents of AYA (between 15 and 25 years old), who agreed to participate. The AYA were both treated in the Medical Oncology Department or in the Paediatric Oncology Department. We conducted individual interviews between October 2005 and April 2006. We met both parents of 17 families, while the other 4 families were composed of one father and 3 mothers. The age of participants varied from 30 to 60 years. The ethics committee of our institution as well as a National Ethic Board gave approval for this research. The researcher in charge of this study and conducting the interviews was a clinician trained in cancer and bereavement research, but external from the Medical Oncology Department and the Paediatric Oncology one. 


\section{Baseline characteristics of families}

\begin{tabular}{ll}
\hline 21 Families & 17 parents (mother \& father) \\
& 1 father \\
& 3 mothers \\
& $43.2 \mathrm{yr}$ \\
Age (years) & 20 \\
Time since death (months) & $18.3 \mathrm{yr}$ \\
Age of deceased children 13 boys 8 girls & SD: 15 - 25 \\
& Ewing sarcoma: $7(33.3 \%)$ \\
& Osteosarcoma: $6(28.6 \%)$ \\
& Rhabdomyosarcoma: $3(14.3 \%)$ \\
& Chondosarcoma: $1(4.7 \%)$ \\
Types of cancer & Neuroblastoma: $1(4.7 \%)$ \\
& Malignant temporal tumour: $1(4.7 \%)$ \\
& Neuroectodermal tumour: $1(4.7 \%) ;$ \\
& Pulmonary adenocarcinoma: $1(4.7 \%)$ \\
& 2 \\
\hline
\end{tabular}

\subsection{Procedure}

We contacted parents by letter signed by the attending physician. Each family was able to refuse the interview by sending an E-mail or by contacting us by phone upon reception of this letter. In the absence of any sign of refusal during the two weeks after sending the letter, the families were contacted by phone by the investigating psychologist to present the protocol and ensure that they did indeed want to participate. When the family agreed to participate, they have to sign a written consent which informed them about the study and the possibility to retract anytime, and then, a semi-structured interview was held in the psycho-oncology unit of the Curie Institute. This interview was recorded and then retranscribed for analysis. Existence of mental disorders (assessed through DSM-IV-TR) was a non-inclusion criterion for participation.

\subsection{Instrument}

The semi-structured interview we used was created especially for this research. To our knowledge, and after a review of the literature, no survey was particularly dedicated to assess needs and supportive care expectations among bereaved parents of AYA through cancer. The interview was the result of a brain-storming session among clinicians from different backgrounds (one medical oncologist, one paediatrician, one psychiatrist and three psychologists) experienced in palliative and supportive care for young patients with cancer. It allows us to clarify the needs of parents who have to cope with this type of situation. We paid a particular attention to formulate questions that were not too disturbing for the participants. The researcher adopted an empathic and supportive attitude towards the participants. A psychological support was offered when acute emotional distress was expressed. But no parents turned toward the psychology unit of the Institute, arguing that they already benefited from an individual psychological support.

The questions asked during the interview were the following:

1) What did you consider to be important for you at the end of your child's life? After his/her death?

2) Has your need(s) been fulfilled?

3) What kind of needs did you feel were not fulfilled and why?

On average, the interviews lasted an hour.

Both a "theoretical" or deductive approach and a thematic or inductive approach were used, based on the Grounded Theory (Charmaz, 2005; Guillemette, 2006; Strauss \& Corbin, 1998) a systematic and standardized method of semantic data analysis. An inductive approach means that the themes identified are strongly linked to the data themselves (Rice \& Ezzy, 1999).

The main researcher (S) who conducted the interviews analysed the data with a second investigator (C). Regular meetings and joint sessions regarding coding, label process and analyse ensure inter ratter reliability. The coding-concept development process was defined as follows: main needs were defined as those emerging in 
more than half of all interviews, and appearing in the parents' answers to the first question (What did you consider to be important for you at the end of your child's life? After his/her death?).

\section{Results}

\subsection{Evocation of Needs: Parents' Perspectives}

One of the main needs highlighted by parents was psychological support during the terminal phase of the child's disease and after the child's death. Indeed 95\% mentioned it. While the 21 parents interviewed spoke about the importance of psychological care ("We need psychological support during the dying of our son [Did you ask for professional help?] No, we were too busy..."), the results showed that no family attended a bereavement group and that only 3 out of 21 (14\%) families consulted a mental health-care professional before and after the child's death (Figure 1).

Two out of the three parents who consulted a mental health-care professional had their child treated in the Medical Oncology Department while the children of the other families were treated in the Paediatric Oncology Department. We noticed no differences between those who consulted and those who did not regarding age, gender or educational levels. However, the 3 parents who consulted a mental health-care professional lived in a big city where more specialists were available. Only 1 out of the 3 parents who consulted a professional presented a mental disorder (complicated grief, diagnosed by the mental-health professional). It is important to notice that expression of psychological support and/or of friendly/familial support emerge spontaneously.

\subsection{What Kind of Support}

One of the main needs underlined by parents was to be listened to and to be understood by the medical team. (97\%) According to their retrospective experience, it seemed that this need was better fulfilled in the Paediatric Oncology Department than it was in the Medical Oncology Department. Indeed, none of parents whose the child was treated in Paediatric Oncology reported any lack of listening. However, as the other parents, they emphasized this point as one of their main needs. All the parents reported having received a great deal of support outside the hospital from their family and/or their friends. They even reported it as their principal means of support $(95 \%)$. Indeed, parents underlined the fact that support from family and friends was most often maintained over time, while such was not the case for the support received from the hospital.

They also insisted on the need for more information at the end of their children's lives including information which was difficult to hear, while their position was quite ambivalent since they sometimes avoided finding out too much about their children's outcome. Finally, all parents with several children emphasized the need for formal grief support for siblings ("Finally my husband and I go help each other to face the death of our daughter... We feel bad but it's normal in this situation... Actually, I think that her brother is really suffering and that he needs professional help but he doesn't agree... He's not speaking about his sister death...”). This reported need is really interesting to investigate and could be associated with former ambivalence. We can suggest that this movement is linked to a frequent tendency among bereaved people: it is easier to seek help for others (for instance children) than for oneself. Some authors also suggested that behind request for helping one's children, there is in fact a request for oneself (Meert et al., 2008). The need for grief support for siblings, expressed by parents, was based on anxious anticipation of impending psychological distress. When asked about actual psychic difficulties, parents reported no symptoms or troubles.

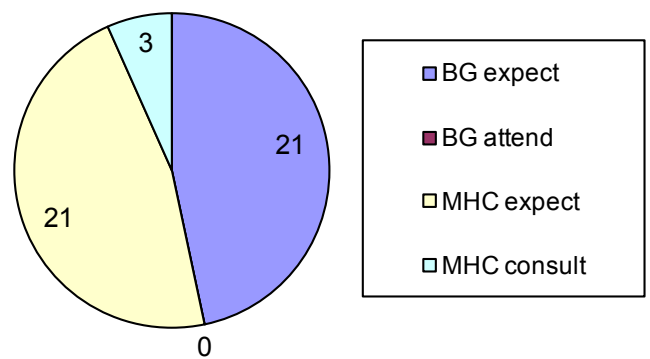

Figure 1. Proportion of families expectation and attendance in bereavement groups and mental health care consultations. 


\subsection{Evolution of Needs}

Importantly, we noticed no differences regarding the needs expressed by parents before and after their child's death. Moreover, no differences were observed according to the culture and socio-economic levels of the parents. Thus, the needs of parents did not seem to differ as a function of these variables. This stability of needs in time could be a result of the particular experience of time for bereaved people. Indeed they frequently report impressions that time is locked or frozen since death of their loved one, and they need time to modify their needs and expectations.

\section{Discussion}

One of the most surprising results of our study is the discrepancy which exists between the needs reported and the number of parents who actually consult a mental health-care professional or join a bereavement group. This gap is already known, but poorly investigated, for other bereft population. For instance it has been established that many bereaved spouses report that psychological support could have helped them, while they did not seek such a support, or even they refused it when it was offered to them (Valdimarsdóttir et al., 2005). This discrepancy could be explained by difficulties for identifying one's own psychic distress, and by common representations among bereaved people, as: "I feel so bad and helpless, but it's normal, I lost my child, and this is not a psychologist/psychiatrist who can help me". Although there is a lack of knowledge about this clinical observation, we can argue that parents have specific psychological needs, the earliest of which are not psychotherapeutic needs. Authors demonstrated that $50 \%$ of the bereaved families spontaneously contact the medical team that took care of their children (Clerici et al., 2006).

Parents expressed the need to be listened to and to be understood by the medical team. The ability to listen to and to provide better comprehension for those suffering cannot be acquired at school or in books. It requires experience, maturity and generosity. Experience and maturity come with time, provided that young nurses and physicians have such time to develop them before they are discouraged by the amount of work and the emotional challenge of being daily confronted with death. We think that it is essential that this time is made available for clinicians to allow them to take care of the needs of young dying patients and their families. Parents who have to cope with the death of a child just need the attentive ear of someone who is able to listen to them without judgment or pity. This listening could come from any of the caregivers and is not only reserved for mental health professionals. In our study, it seems that the most important support was actually provided outside the hospital by family and friends. Whoever gives it and whenever it is given, the patient and his/her family deserve such attention. As some studies show, in the long term, sharing one's emotional burden with others facilitates the grieving process (Kreicbergs et al., 2007).

Our results also demonstrated that the need for information is variable and this fact must be understood by the medical team in order that they respect the psychic defences of each individual. Therefore, when parents do not seem to clearly understand the message that physicians or nurses want to transmit them, this should not be a cause for worry. It is part of the coping process developed by parents and should not to be confused with denial which refers to a pathological rejection of reality. Interestingly, authors established a difference between intellectual and emotional awareness (Valdimarsdóttir et al., 2007). Logically, the period of emotional awareness is always longer but nonetheless, it should not be too much longer because of the risk of developing psychological disorders (Valdimarsdóttir et al., 2007). Therefore, as health professionals, we have to be vigilant in order to distinguish the period necessary for emotional integration from the inability for parents to integrate the child's death. In the same way, literature regarding preparation for the death of a loved one emphasizes a frequent difference between cognitive comprehension of the fatal prognosis (comparable to intellectual awareness) and the feeling of being prepared to the death (that is emotional awareness) (Hebert et al., 2006). As a consequence of this difference, this emotional preparedness is not only depending on medical information.

The need for formal grief support for siblings, reported by all parents with several children, was also highlighted in literature (DeCinque et al., 2006). However, our experience shows that few siblings of a deceased child ask for psychological support. Parents have to encourage their children to ask for psychological help and in many cases, the needs of adults and children do not have the same evolution and parents have difficulties evaluating the siblings' psychological needs and outcomes. We have observed that sometimes, parents speak about themselves when they ask for psychological help for their children, and we should think about different ways to help parents and their children. Do they need individual or familial approaches to work on the changes in famil- 
ial functioning after the child's death? Do they need group therapy to share emotion and express their grief? It seems very important to take the time to make a clear evaluation of the needs of parents and children (and of the family as a system), then to help them choose the response most adapted to their needs. A relevant way to improve this screening of distress among siblings could be support for parents through educational groups on how to support their other children

The culture and socio-economic levels of these families did not influence the needs of the parents, although we know that culture influences the way illness and death are understood (Hass \& Walter, 2007). In this study, it should be noted that only 4 families were not caucasian. Moreover, all of them had lived in France for several decades and our finding could be the result of their cultural assimilation. Finally, this result could reflect the difference between personal beliefs about death and illness and personal needs during a child's illness and after his death. Thus, in this perspective, parents' needs might not be influenced by cultural background very much.

In the future, it would be useful to investigate the needs of each parent separately in order to better distinguish them, since the risk of developing mental disorders after a child's death seems to vary as a function of gender (Li et al., 2005).

This study identifies particular needs of parents which have lost a child due to cancer, but it contains some bias. The retrospective method is a bias to identify clearly the evolution of needs during the terminal illness and after the death. A prospective investigation with several times of interviews (during terminal illness and after the death for example) should provide more information about parental needs. Concerning the siblings, it will be very interesting to investigate their needs by interviews and to compare it with parental evaluation. We also think that familial approach and investigation can be the way to develop family centred interventions to facilitate changes in familial functioning after the death of the child.

\section{Conclusion}

Our study clearly demonstrates that parents who have lost a child suffering from cancer have particular needs and therefore deserve special attention. Identifying their personal needs is vital in order to improve the assistance that all of us can provide for them.

\section{Acknowledgements}

This research was financed by the Fondation de France.

The authors would like to thank Dr. L. Mignot, Head of the Department of Medical Oncology and Dr. J. Michon, Head of the Department of Paediatric Oncology, for their support during this research, as well as all pediatricians of the Curie Institute, Department of Paediatric Oncology for their collaboration during this study.

\section{Conflict of Interest}

The authors declare that there is no conflict of interest in this research.

\section{References}

Barrera, M., O’Connor, K., D’Agostino, N. M., Spencer, L., Nicholas, D., Jovcevska, V., Tallet, S., \& Schneiderman, G. (2009). Early Parental Adjustment and Bereavement after Childhood Cancer Death. Death Studies, 33, 497-520. http://dx.doi.org/10.1080/07481180902961153

Beck, A., \& Konnert, C. (2007). Ethical Issues in the Study of Bereavement: The Opinions of Bereaved Adults. Death Studies, 31, 783-799. http://dx.doi.org/10.1080/07481180701537220

Charmaz, K. (2005). Grounded Theory in the 21 st Century. In N. K. DansDenzin, \& Y. S. Lincoln (Eds.), The Sage Handbook of Qualitative Research (3rd ed., pp. 507-535). Thousand Oaks, CA: Sage.

Christ, G. H., Bonanno, G. A., Malkinson, R., \& Rubin, S. (2003). Bereavement Experiences after the Death of a Child. In M. J. Field, \& R. E. Behrman (Eds.), When Children Die: Improving Palliative and End-of-Life for Children and Their Families (pp. 553-579). Washington DC: National Academy Press.

Clerici, C. A., Ferrari, A., Massimino, M., Luksch, R., Cefalo, G., \& Terenziani, M. (2006). Assistance to Parents Who Have Lost Their Child with Cancer. Tumori, 92, 306-310.

D’Agostino, N. M., Berlin-Romalis, D., Jovcevska, V., \& Barrera, M. (2008). Bereaved Parents' Perspectives on Their Needs. Palliative and Supportive Care, 6, 33-41. http://dx.doi.org/10.1017/S1478951508000060 
DeCinque, N., Monterosso, L., Dadd, G., Sidhu, R., Macpherson, R., \& Aoun, S. (2006). Bereavement Support for Families Following the Death of a Child from Cancer: Experience of Bereaved Parents. Journal of Psychosocial Oncology, 24, 6583. http://dx.doi.org/10.1300/J077v24n02_05

Dyregrov, K. (2004). Bereaved Parents' Experience of Research Participation. Social Science \& Medicine, 58, 391-400. http://dx.doi.org/10.1016/S0277-9536(03)00205-3

Goodenough, B., Drew, D., Higgins, S., \& Trethewie, S. (2004). Bereavement Outcomes for Parents Who Lose a Child to Cancer: Are Place of Death and Sex of Parent Associated with Differences in Psychological Functioning? Psycho-Oncology, 13, 779-791. http://dx.doi.org/10.1002/pon.795

Guillemette, F. (2006). L’Approche de la Grounded Theory, Pour Innover? Recherches Qualitatives, 26, 32-50.

Hass, J. K., \& Walter, T. (2007). Parental Grief in Three Societies: Networks and Religion as Social Supports in Mourning. Omega, 54, 179-198. http://dx.doi.org/10.2190/023P-2J03-8511-561R

Hebert, R. S., Prigerson, H. G., Schulz, R., \& Arnold, R. M. (2006). Preparing Caregivers for the Death of a Loved One: A Theoretical Framework and Suggestions for Future Research. Journal of Palliative Medicine, 9, 1164-1171.

http://dx.doi.org/10.1089/jpm.2006.9.1164

Hynson, J., Aroni, R., Bauld, C., \& Sawyer, S. M. (2006). Research with Bereaved Parents: A Question of How Not Why. Palliative Medicine, 20, 805-811. http://dx.doi.org/10.1177/0269216306072349

Kavanaugh, K., Trier, D., \& Korzec, M. (2004). Social Support Following Perinatal Loss. Journal of Family Nursing, 10, 70 92. http://dx.doi.org/10.1177/1074840703260905

Kreicbergs, U. C., Lannen, P., Onelov, E., \& Wolfe, J. (2007). Parental Grief after Losing a Child to Cancer: Impact of Professional and Social Support on Long-Term Outcomes. Journal of Clinical Oncology, 25, 3307-3312. http://dx.doi.org/10.1200/JCO.2006.10.0743

Li, J., Laursen, T. M., Precht, D. H., Olsen, J., \& Mortensen, P. B. (2005). Hospitalization for Mental Illness among Parents after the Death of a Child. New England Journal of Medicine, 352, 1190-1196. http://dx.doi.org/10.1056/NEJMoa033160

Meert, K., Eggly, S., Dean, J., Pollack, M., Zimmerman, J., Anand, K., Newth, C., Willson, D., \& Nicholson, C. (2008). Ethical and Logistical Considerations of Multicenter Parental Bereavement Research. Journal of Palliative Medicine, 11, 444-450. http://dx.doi.org/10.1089/jpm.2007.0120

Montel, S., Laurence, V., Copel, L., Pacquement, H., \& Flahault, C. (2008). Place of Death of Adolescent and Young Adults with Cancer: First Study in a French Population. Palliative and Supportive Care, 7, 27-35. http://dx.doi.org/10.1017/S1478951509000054

Monterosso, L., \& Kristjanson, L. J. (2008). Supportive and Palliative Care Needs of Families of Children Who Die from Cancer: An Australian Study. Palliative Medicine, 22, 59-69. http://dx.doi.org/10.1177/0269216307084608

Rice, P. L., \& Ezzy, D. (1999). Qualitative Research Methods: A Health Focus. South Melbourne (Australia): Oxford University Press.

Strauss, A. L., \& Corbin, J. (1998). Basics of Qualitative Research (2nd ed.). Thousand Oaks, CA: Sage.

Valdimarsdóttir, U., Helgason, A. R., Fürst, C. J., Adolfsson, J., \& Steineck, G. (2005). Need for and Access to Bereavement Support after Loss of a Husband to Urologic Cancers: A Nationwide Follow-Up of Swedish Widows. Scandinavian Journal of Urology and Nephrology, 39, 271-276. http://dx.doi.org/10.1080/00365590510031273

Valdimarsdóttir, U., Kreicbergs, U., Hauksdóttir, A., Hunt, H., Onelöv, E., \& Henter, J. I. (2007). Parents' Intellectual and Emotional Awareness of Their Child's Impending Death to Cancer: A Population-Based Long-Term Follow-Up Study. Lancet Oncology, 8, 706-714. http://dx.doi.org/10.1016/S1470-2045(07)70209-7 